\title{
Verzeichnis der einzelnen Briefwechsel
}

Für den Zeitraum von Januar 1811 bis Juni 1813 lassen sich insgesamt 370 Briefe, davon 149 von Schleiermacher und 221 an ibn, belegen. Davon sind 101 Briefe, also ein gutes Viertel erschlossen, 269 sind in Form von Handschriften, Abschriften oder Drucken überliefert. Neben den Hinweisen aus Briefen sind auch Schleiermachers Tageskalender eine wichtige Quelle für die Erschließung von Briefen, insofern Schleiermacher hier auch Brief Ein- und Ausgänge verzeichnete. Schleiermacher begann seine Tageskalender 1808 und fübrte sie zum Teil mit großen Unterbrechungen bis kurz vor seinem Tod 1834 fort. Für den hier dokumentierten Zeitraum gibt es jedoch lediglich einen Tageskalender für das Jahre 1811.

Die in diesem Band angeführten Briefe enthalten Schleiermachers persönliche Korrespondenz für diesen Zeitraum sowie ausgewählte Briefe der amtlichen Korrespondenz. Insgesamt treten von Januar 1811 bis Juli 1813100 namentlich genannte oder ermittelte Korrespondenzpartner in Erscheinung, darunter ein Viertel Frauen (23) und 4 nur per Nachnabme identifizierte Personen. Darüber hinaus sind 23 Briefe von nicht ermittelten Personen zu verzeichnen ( 3 darunter von derselben Person). Die hier dokumentierte amtliche Korrespondenz umfasst insgesamt 14 Briefe. Die drei umfänglichsten Briefwechsel führt Schleiermacher in diesen Zeitraum mit seiner Frau Henriette Schleiermacher (36 Briefe), seiner Schwester Charlotte Schleiermacher (26 Briefe) und seinem Freund Joachim Christian Gaß (20 Briefe).

Insgesamt werden in diesem Briefband 188 Briefe zum ersten Mal gedruckt (35 von Schleiermacher, 153 an ihn), 41 bereits gedruckte Briefe werden erstmals vollständig wiedergegeben (darunter 24 von Schleiermacher und 17 an ihn). Erstmals textkritisch wiedergegeben werden insgesamt 40 Briefe, darunter 14 an und 26 von Schleiermacher.

Die folgende Liste ist alphabethisch nach Korrespondenzpartner undpartnerin sortiert. Ihr folgt eine Liste der Korrespondenz mit nicht identifizierten Personen sowie der amtlichen Korrespondenz (die, sofern namentlich bekannt, auch in die alphabetische Reihenfolge integriert sind). Im Anschluss an die amtliche Korrespondenz sind die Nachträge zu den Briefbänden KGA V/10 und 11 angeführt. 
Das *vor der Briefnummer bezeichnet erschlossene Briefe.

Ammon, Christoph Friedrich von (1766-1849)

Professor der Theologie in Erlangen

3572. Von Christoph Friedrich Ammon.

Erlangen, Sonnabend, 12.1.1811

3575. Von Christoph Friedrich Ammon. Erlangen, Dienstag, 15.1.1811 ............ 29

*3582. An Christoph Friedrich Ammon. Berlin, Dienstag, 22.1.1811 ............... 39

*3589. An Christoph Friedrich Ammon. Berlin, Sonnabend, 9.2.1811 ............. 46

Arnold, Friedrich Wilhelm

Bekannter Schleiermachers in Stolp

*3741. An Friedrich Wilhelm Arnold.

Berlin, vor dem 15.2.1812 ............. 236

3745. Von Friedrich Wilhelm Arnold.

Stolp, Sonnabend, 15.2.1812 ............ 237

3776. Von Friedrich Wilhelm Arnold.

Stolp, Sonnabend, 18.4.1812 ............ 271

*3777. An Friedrich Wilhelm Arnold.

Berlin, Freitag, 24.4.1812 ............ 272

Bachmann, Karl Friedrich (1785-1855)

Philosoph und Mineraloge

3561. Von Karl Friedrich Bachmann.

Jena, Donnerstag, 3.1.1811 ............. 3

Bartholdy (Bartoldi), Georg Wilhelm (1765-1815)

seit 1797 Lehrer am Gymnasium in Stettin

*3585. An Georg Wilhelm Bartholdy.

Berlin, wohl Januar 1811 ............... 44

3608. Von Georg Wilhelm Bartholdy.

Stettin, Mittwoch, 27.3.1811 ............ 71

3657. Von Georg Wilhelm Bartholdy.

Stettin, Mittwoch, 31.7.1811 ............ 125

*3666. An Georg Wilhelm Bartholdy.

Berlin, vor dem 25.8.1811 ............. 133 
3667. Von Georg Wilhelm Bartholdy.

Stettin, Sonntag, 25.8.1811 ............... 133

3713. Von Georg Wilhelm Bartholdy.

Stettin, Donnerstag, 12.12.1811 ............ 196

Bekker (Becker), August Immanuel (1785-1871)

Altphilologe, Schleiermacherhörer, Professor in Berlin seit 1810

*3783. An Immanuel Bekker.

Wohl Berlin, vor dem 15.6.1812 ............ 276

3787. Von Immanuel Bekker.

Paris, Sonntag, 5.7.1812 ................. 280

*3796. An Immanuel Bekker.

Berlin, Sonnabend, 1.8 .1812 ............... 291

Bellermann, Johann Joachim (1754-1842)

Kirchenhistoriker, Philosoph und Pädagoge, seit 1804 Direktor des Gymnasiums zum Grauen Kloster in Berlin

3791. An Johann Joachim Bellermann.

Berlin, Mittwoch, 22.7.1812 ............. 288

Bertuch, Friedrich Justin (1747-1822)

Verleger und Schriftsteller, Gründer der Allgemeinen Literatur-Zeitung (ALZ)

3734. Von Friedrich Justin Bertuch.

Weimar, Freitag, 24.1.1812 .............. 219

Beyme, Karl Friedrich von (1765-1838)

preußischer Staatsmann

*3855. An Karl Friedrich von Beyme.

Berlin, Donnerstag, 13.5.1813 oder früher ...... 366

Biester, Johann Erich (1749-1816)

Aufklärer; Bibliothekar und Mitglied der Akademie der Wissenschaften in Berlin

3809. An Johann Erich Biester.

Berlin, Sonnabend, 19.10.1811 oder Montag,

19.10 .1812 ......................... 301 


\section{Binder}

3695. Von Binder (?)

Berlin, Sonnabend, 19.10.1811

\section{Birnbach, Caroline}

3697. Von Caroline Birnbach.

Blanc, Ludwig Gottried (1781-1866)

1806 französisch-reformierter Prediger in Halle, ab 1809 dort Domprediger, 1811-13 in Haft

3564. Von Ludwig Gottfried Blanc.

Halle, Sonnabend, 5.1.1811 .............. 6

3677. Von Ludwig Gottfried Blanc.

Halle, Sommer 1811 .................. 141

Boyen, Hermann von (1771-1848)

preußischer Offizier

3857. An Hermann von Boyen.

Berlin, Donnerstag, 13.5.1813 ........... 368

3860. Von Hermann von Boyen.

Berlin, Freitag, $14.5 .1813 \quad \ldots \ldots \ldots \ldots \ldots \ldots \ldots$

Brinckmann (schwed. Brinkman), Carl Gustav von (1764-1847)

Dichter und schwedischer Diplomat, enger Freund Schleiermachers

3785. An Carl Gustav von Brinckmann.

Berlin, Sonnabend, 4.7.1812 ............ 276

\section{Cummerow, Charlotte}

geb. Israel, Freundin Schleiermachers, wohnhaft in Stralsund

3577. Von Charlotte Cummerow.

Stralsund, Mittwoch, 16.1.1811 .......... 31

3789. Von Charlotte Cummerow.

Kopenhagen, Mittwoch, 15.7.1812 _....... 282

Dippold, Hans Karl (1783-1811)

ab 1810 Professor der Geschichte und Geographie am akademischen Gymnasium in Danzig

3656. Von Hans Karl Dippold.

Danzig, Dienstag, 30.7.1811 ............. 123 
Dohna-Schlobitten, Alexander Graf zu (1771-1831)

preußischer Staatsmann, 1808-1810 preußischer Innenminister, Freund Schleiermachers

3574. An Alexander Graf zu Dohna-Schlobitten.

Berlin, Montag, 14.1.1811 ................ 27

*3758. Von Alexander Graf zu Dohna-Schlobitten.

Vor Mitte März 1812 .................... 257

3759. An Alexander Graf zu Dohna-Schlobitten.

Berlin, Mitte März 1812 ................. 258

3820. An Alexander Graf zu Dohna-Schlobitten.

Berlin, Sonnabend, 2.1.1813 ............... 319

*3837. Von Alexander Graf zu Dohna-Schlobitten.

Vor dem 23.3.1813 ..................... 345

3838. An Alexander Graf zu Dohna-Schlobitten.

Berlin, Dienstag, 23.3. bis Sonnabend, 27.3.1813 . . 345

3848. An Alexander Graf zu Dohna-Schlobitten (auch von

Henriette Herz).

Berlin, Sonnabend, 17.4.1813 .............. 355

*3863. An Alexander Graf zu Dohna-Schlobitten.

Berlin, Sonnabend, 15.5.1813 .............. 375

Dohna-Schlobitten, Wilhelm Graf zu (1773-1845)

Bruder des Alexander Graf zu Dohna-Schlobitten

3786. An Wilhelm Graf zu Dohna-Schlobitten.

Berlin, Sonntag, 5.7.1812 ................ 279

*3797. Von Wilhelm Graf zu Dohna-Schlobitten.

Sonnabend, 1.8 .1812 .................. 291

Dräseke, Johann Heinrich Bernhard (1774-1849)

1804 Pfarrer an St. Georg auf dem Berge bei Ratzeburg

*3842. Von Johann Heinrich Bernhard Dräseke.

März 1813

Fischer, Ernst Gottfried (1754-1831)

Professor der Mathematik und Physik am Gymnasium zum Granen Kloster in Berlin; ab 1810 auch außerordentlicher Professor an der Berliner Universität

3660. Von Ernst Gottfried Fischer.

Berlin, Anfang August 1811 oder früher ........ 128 
Frommann, Karl Friedrich Ernst (1765-1837)

Verleger und Buchhändler in Jena

3587. Von Friedrich Frommann.

Jena, Donnerstag, 7.2.1811 ............ 45

*3601. An Friedrich Frommann.

Berlin, Sonnabend, 16.3.1811 ............. 61

*3628. An Friedrich Frommann.

April 1811 .......................... 89

3637. Von Friedrich Frommann.

Leipzig, Freitag, 24.5.1811 ............. 102

Froriep, Ludwig Friedrich von (1779-1847)

Arzt, seit 1804 Professor der Medizin in Halle

3714. An Ludwig Friedrich von Froriep.

Berlin, Sonnabend, 14.12.1811 ............ 198

*3733. Von Ludwig Friedrich von Froriep.

Vor dem 23.1.1812 .................... 219

Furchau, Adolf Friedrich (1787-1868)

Dichter, Hauslehrer im Hause Kathen

3566. Von Adolf Friedrich Furchau.

Götemitz, Montag, 7.1.1811 ............ 14

3635. An Adolf Friedrich Furchau.

Vor dem 22.5.1811 ..................... 99

3636. Von Adolf Friedrich Furchau.

Götemitz, Mittwoch, 22.5.1811 ............ 100

Gaß, Joachim Christian (1766-1831)

ab 1810 Kirchenrat und ab 1811 Professor der Theologie in Breslau, Freund Schleiermachers

3567. Von Joachim Christian Gaß.

Breslau, Montag, 7.1.1811 ............ 15

3595. Von Joachim Christian Gaß.

Breslau, Mittwoch, 20.2.1811 ........... 53

3613. Von Joachim Christian Gaß.

Breslau, Donnerstag, 4.4.1811 .......... 75

3620. Von Joachim Christian Gaß.

Breslau, Mittwoch, 17.4.1811 ............. 79

3630. An Joachim Christian Gaß.

Berlin, Sonnabend, 11.5.1811 ........... 90 
3634. Von Joachim Christian Gaß.

Breslau, Dienstag, 21.5.1811 .............. 96

3645. Von Joachim Christian Gaß.

Breslau, Montag, 10.6.1811 ............... 109

3646. An Joachim Christian Gaß.

Berlin, Montag, 17.6.1811 ................ 110

3682. Von Joachim Christian Gaß.

Breslau, Montag, 16.9.1811 ............... 149

3696. An Joachim Christian Gaß.

Berlin, Mittwoch, 23.10.1811 ............. 172

3710. Von Joachim Christian Gaß.

Breslau, Sonntag, 8.12.1811 ............... 189

3724. Von Joachim Christian Gaß. 1811 .............................. 208

3728. An Joachim Christian Gaß.

Berlin, um den 5.1. bis Mittwoch, 12.2.1812 ..... 211

3749. Von Joachim Christian Gaß.

Breslau, Sonntag, 23.2.1812 ............... 241

3810. An Joachim Christian Gaß.

Berlin, Sonnabend, 24.10 .1812 ............. 302

3811. Von Joachim Christian Gaß.

Breslau, Mittwoch, 28.10.1812 .............. 303

3816. An Joachim Christian Gaß.

Berlin, Sonnabend, 21.11.1812 .............. 313

3823. An Joachim Christian Gaß.

Berlin, Sonntag, 24.1.1813 ................ 324

3836. Von Joachim Christian Gaß.

Breslau, Donnerstag, 11.3. bis Sonntag, 14.3.1813 . 340

3929. Von Joachim Christian Gaß.

Reinerz, Mittwoch, 30.6.1813 .............. 474

Giesebrecht, Karl Ludwig (1782-1832)

Theologe und Altphilologe, seit 1805 Professor am Pädagogium in Bremen

3725. Von Karl Heinrich Ludwig Giesebrecht.

Bremen, Mittwoch, 1.1.1812 .............. 208 
XLVIII Verzeichnis der einzelnen Briefwechsel

Grabl, Carl Friedrich (1765-1834)

Küster an der Dreifaltigkeitskirche, Lehrer und Kantor

3611. Von Carl Friedrich Grabl.

Berlin, Montag, 1.4.1811 ............ 74

Ha(b)ne, Amalie

lebte im Sagarder Pfarrhaus auf Rügen, wohl Schwester der Philippine Schwarz, geb. Ha(b)ne

*3829. Von Amalie Ha(b)ne (auch an Henriette (Jette) Schleiermacher).

Wobl Sagard, um den 9.2.1813 ............ 328

Hardenberg, Karl August Freiherr von (1750-1822)

preußischer Staatsmann und Reformer, seit 1810 Staatskanzler

3731. An Karl August Freiherr von Hardenberg (auch von der

Sektion des öffentlichen Unterrichts).

Berlin, Montag, 13.1.1812 .............. 218

3813. An Karl August Freiherr von Hardenberg (auch von der

Sektion des öffentlichen Unterrichts).

Berlin, Montag, 9.11.1812 .............. 308

3839. Von Karl August Freiherr von Hardenberg (auch an Barthold Georg Niebuhr).

Berlin, Donnerstag, 25.3.1813

Hecker, Andreas Jakob (1746-1819)

Prediger an der Dreifaltigkeitskirche in Berlin, Oberkonsistorial- und Oberschulrat

3674. An Andreas Jakob Hecker.

Berlin, Sonnabend, 31.8.1811 ........... 137

3735. An Andreas Jakob Hecker.

Berlin, Sonnabend, 25.1.1812 ............ 220

Herz, Henriette (Jette) (1764-1847)

geb. de Lemos, Salonnière, sehr gute Freundin F. Schleiermachers

*3866. Von Henriette Herz.

Breslau, vor dem 16.5.1813 .............. 376

*3904. Von Henriette Herz.

Neiße, vor dem 10.6.1813 ..............446 
Herzberg, Friedrich (1763-1822)

1792-1822 zweiter lutherischer Prediger an der Dreifaltigkeitskirche Berlin

*3726. Von Friedrich Herzberg.

Berlin, Mittwoch, 1.1.1812 .............. 210

3730. An Friedrich Herzberg (auch von der Sektion des Unterrichts). Berlin, Montag, 13.1.1812 ............. 217

Hering, Christlieb Benjamin (gest. 1827)

Kaufmann in Stolp

3581. Von Christlieb Benjamin Hering.

Stolp, Sonnabend, 19.1.1811 .......... 37

Hochwächter, Christoph Ludwig von (1769-1838)

Mann der Pauline von Hochwächter, Herr auf Groß und Klein Miltzow 3853. Von Christoph Ludwig Hochwächter.

Milzow, Sonnabend, 1.5.1813 .................. 365

Hochwächter, Pauline von (1776-1859)

geb. von Müblenfels, Schwester der Henriette (Jette) Schleiermacher

3930. Von Pauline Hochwächter.

Milzow, zweite Hälfte Juni 1813 .........4 475

Hufeland, Christoph Wilhelm (1762-1836)

seit 1801 Leiter der Berliner Charité und Leibarzt der Königin Luise von Preußen

*3649. An Christoph Wilhelm Hufeland.

Berlin, vor dem 30.6.1811 .............. 115

3650. Von Christoph Wilhelm Hufeland.

Berlin, Sonntag, 30.6.1811 ............ 115

Hoffmann, Johann Gottfried (1765-1847)

Nationalökonom, 1810-1814 Professor an der Berliner Universität

*3768. An Johann Gottfried Hoffmann.

Berlin, März 1812 .................... 265

*3770. Von Johann Gottfried Hoffmann.

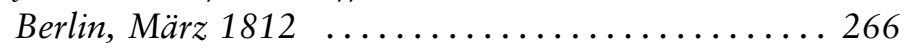


Israel, Friederike (1777-1829)

geb. Stenzler, Freundin Schleiermachers

*3868. An Friederike Israel.

Berlin, Sonntag, 16.5.1813

Kathen, Charlotte von (1777-1850)

geb. von Müblenfels, Freundin F. Schleiermachers, Schwester von

Henriette (Jette) Schleiermacher, Salonnière auf Rügen

*3597. Von Charlotte von Kathen.

Januar/Februar $1811 \ldots \ldots \ldots \ldots \ldots \ldots \ldots . \ldots 58$

3598. An Charlotte von Kathen.

Berlin, Donnerstag, 7.3.1811 .............. 59

*3669. An Charlotte von Kathen.

Vor dem 26.8 .1811 ..................... 134

*3671. Von Charlotte von Kathen.

Vor dem 26.8.1811 ...................... 135

3676. An Charlotte von Kathen.

Berlin, Sonntag, 1.9.1811 ................ 139

3740. An Charlotte von Kathen.

Berlin, Donnerstag, 13.2. bis Sonnabend, 15.2.1812 .......................... 234

*3800. Von Charlotte von Kathen und Luise von Willich.

Vor dem 11.8.1812 ....................... 292

3856. An Charlotte von Kathen.

Berlin, Donnerstag, 13.5.1813 ............. 366

3895. An Charlotte von Kathen.

Berlin, Donnerstag, 3.6.1813 ............. 427

*3917. Von Charlotte von Kathen.

Vor dem 16.6.1813 ..................... 462

3918. An Charlotte von Kathen.

Berlin, Donnerstag, 17.6.1813 ............. 463

*3923. Von Charlotte von Kathen.

23.6.1813 oder früher ................... 468

Klischnig, Karl Friedrich (1766 - gest. vor 1825)

Schriftsteller und Übersetzer

3643. Von Karl Friedrich Klischnig.

Berlin, Anfang Juni 1811 oder früher .......... 108 
Körner, Christian Gottfried (1756-1831)

Schriftsteller und Jurist, Vater des Theodor Körner

3610. Von Christian Gottfried Körner.

Dresden, Sonntag, 31.3.1811 ........... 73

3618. An Christian Gottfried Körner.

Berlin, vor dem 14.4.1811 .............. 78

3619. Von Christian Gottfried Körner.

Dresden, Sonntag, 14.4.1811 ........... 78

Konopak, Christian Gottlieb (1767-1841)

1802 Professor der Rechte in Halle, 1807 in Rostock, 1817 in Jena

3568. Von Christian Gottlieb Konopak.

Rostock, Dienstag, 8.1.1811 ............. 16

3586. Von Christian Gottlieb Konopak.

Rostock, Montag, 4.2.1811 ............. 44

*3596. An Christian Gottlieb Konopak.

Wobl Mitte oder Ende Februar 1811 ......... 58

3607. Von Christian Gottlieb Konopak.

Rostock, vor dem 24.3.1811 .............. 69

3652. Von Christian Gottlieb Konopak.

Rostock, Montag, 8.7.1811 ............... 119

*3679. An Christian Gottlieb Konopak.

Berlin, Freitag, 13.9.1811 .............. 146

3688. Von Christian Gottlieb Konopak.

Rostock, Sonntag, 22.9.1811 ............ 157

3794. Von Christian Gottlieb Konopak.

Rostock, vor dem 28.7.1812 ............. 289

*3795. An Christian Gottlieb Konopak.

Berlin, Dienstag, 28.7.1812 ............. 290

3812. Von Christian Gottlieb Konopak.

Rostock, Donnerstag, 29.10.1812 _......... 307

3878. Von Christian Gottlieb Konopak.

Rostock, Donnerstag, 20.5.1813 ......... 392

Kosegarten, Gotthard Ludwig Theobul (1758-1818)

Pfarrer auf Rügen, Schriftsteller

3621. Von Ludwig Kosegarten.

Greifswald, Donnerstag, $18.4 .1811 \ldots \ldots \ldots . \ldots 2$ 
Levi (Levin), Rabel (1771-1833)

Schriftstellerin und Salonnière

3736. An Rabel Levin.

Berlin, Mittwoch, 29.1.1812 _........... 221

Loeffler (Löffler), Josias Friedrich Christian (1752-1816)

seit 1788 Generalsuperintendent in Gotha, Herausgeber des Magazins für Prediger

3602. An Josias Friedrich Christian Loeffler.

Berlin, Sonnabend, 16.3.1811 ............ 61

Marbeineke, Philipp Konrad (1780-1846)

Professor der Theologie in Erlangen, seit 1807 in Heidelberg, 1811 in Berlin

*3588. An Philipp Konrad Marheineke.

Berlin, vor dem 8.2.1811 ............. 46

3591. Von Philipp Konrad Marheineke.

Heidelberg, Sonnabend, 9.2.1811 ......... 47

3699. Von Philipp Konrad Marheineke.

Berlin, Anfang November 1811 oder früher ..... 175

Martini, Christoph David Anton (1761-1815)

evangelischer Theologe, seit 1809 Kreiskirchenrat und Gymnasialprofessor in München

*3599. An Christoph David Anton Martini.

Berlin, Sonnabend, 9.3.1811 ............. 60

3606. Von Christoph David Anton Martini.

München, Freitag, 22.3.1811 ........... 67

*3615. Von Christoph David Anton Martini.

München, um den 5.4.1811 ............. 77

3633. Von Christoph David Anton Martini.

München, Sonntag, 19.5.1811 ........... 95

Marwitz, Alexander von der (1787-1814)

preußischer Offizier, Schleiermacherhörer in Halle, Geliebter der

Henriette (Jette) Schleiermacher

*3846. An Alexander von der Marwitz (auch von Henriette Schleiermacher).

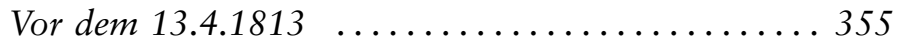


*3847. An Alexander von der Marwitz (auch von Henriette Schleiermacher).

Berlin, Dienstag, 13.4.1813 ............. 355

*3854. Von Alexander von der Marwitz.

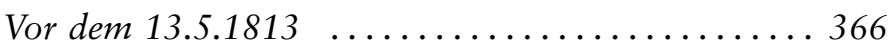

*3864. An Alexander von der Marwitz.

Berlin, Sonnabend, 15.5.1813 ............ 375

*3896. An Alexander von der Marwitz.

Berlin, Donnerstag, 3.6.1813 _..........4 428

Mebmel, Gottlieb Ernst August (1761-1840)

Professor der Philosophie an der Universität Erlangen, Redakteur der Erlanger Literaturzeitung

3698. Von Gottlieb Ernst August Mehmel.

Erlangen, Freitag, 1.11.1811

Metger, Friedrich Severin (1775-1834)

Schleiermachers Nachfolger als reformierter Prediger an der Charité (1802) und in Stolp (1807/08), Schleiermacherhörer in Halle

3570. Von Friedrich Severin Metger.

Stolp, Donnerstag, 10.1.1811 ........... 20

*3603. An Friedrich Severin Metger.

Berlin, Sonnabend, 16.3.1811 ........... 63

3651. Von Friedrich Severin Metger.

Wobl zwischen Ende März und Ende Juni 1811 ... 115

3757. Von Friedrich Severin Metger.

Stolp, Mittwoch, 11.3.1812 ............. 255

Mieth, Gottfried (1765-1834)

Bronzefabrikant und Kirchenvorsteher an der Dreifaltigkeitskirche

3775. Von Gottfried Mieth.

Berlin, Montag, 13.4.1812 ............. 270

Müblenfels, Friedrich von (1778-1852)

Bruder der Henriette (Jette) von Willich, Herr auf Sissow

*3738. An Friedrich von Müblenfels.

Dezember 1811/Januar 1812 ............. 232

3782. Von Friedrich von Müblenfels.

Züssow, Mittwoch, 10.6.1812 ........... 274 
*3825. An Friedrich von Müblenfels.

Januar 1813 oder früber

3828. Von Friedrich von Müblenfels.

Züssow, Montag, 8.2.1813

Müller, Adolph Wilbelm (1784-1811)

Sohn des Wilhelm Christian Müller, Schleiermacherhörer in Halle, seit 1809 praktizierender Arzt in Bremen

*3562. An Adolph Müller.

Berlin, vor dem 4.1.1811

Müller, Elise (1782-1849)

Tochter des Wilhelm Christian Müller, Musikerin

*3716. An Elise Müller.

Berlin, vor dem 17.12.1811 .............. 200

3717. Von Elise Müller.

Bremen, Dienstag, 17.12.1811 ........... 201

Müller, Wilhelm Christian (1752-1831)

Musiker, Schriftsteller, Kantor und Pädagoge in Bremen

3565. Von Wilhelm Christian Müller.

Bremen, Sonnabend, 5.1. bis Sonntag, 6.1.1811 ... 9

3580. An Wilhelm Christian Müller.

Berlin, Sonnabend, 19.1.1811 ............ 34

3584. Von Wilhelm Christian Müller.

Bremen, Montag, 28.1.1811 ............. 40

3592. An Wilhelm Christian Müller.

Berlin, Dienstag, 12.2.1811 ............. 48

3654. Von Wilhelm Christian Müller.

Bremen, Donnerstag, 18.7.1811 ........... 121

3678. Von Wilhelm Christian Müller.

Bremen, Donnerstag, 12.9.1811 ............ 145

3694. Von Wilhelm Christian Müller.

Bremen, Sonntag, 13.10.1811 ............ 171

3818. Von Wilhelm Christian Müller.

Bremen, Mittwoch, 16.12.1812 .......... 317

3833. Von Wilhelm Christian Müller.

Bremen, Donnerstag, 4.3.1813 ........... 336

3905. Von Wilhelm Christian Müller.

Boizenburg, Donnerstag, 10.6.1813 ........ 436 
3913. Von Wilhelm Christian Müller.

Wilsnack, Sonntag, 13.6.1813 .............. 445

Niebubr, Barthold Georg (1776-1831)

Historiker, seit 1810 Professor in Berlin und Mitglied der Berliner Akademie der Wissenschaften

3625. Von Barthold Georg Niebuhr.

Berlin, Montag, 8.4., 22.4. oder 29.4.1811 ..... 85

*3626. An Barthold Georg Niebuhr.

Berlin, wohl Ende April 1811 ............. 87

3627. Von Barthold Georg Niebuhr.

Berlin, wohl Ende April 1811 ............. 87

Nitzsch, Carl Ludwig (1751-1831)

1790 Pastor und Superintendent in Wittenberg, 1817 ebenda Direktor des Predigerseminars

3624. Von Carl Ludwig Nitzsch.

Wittenberg, Sonntag, 28.4.1811 ............ 83

Perthes, Friedrich Christoph (1772-1843)

Buchbändler und Verleger in Hamburg, Neffe des Justus Perthes

3851. An Friedrich Perthes.

Berlin, Dienstag, 27.4.1813 ................ 361

Pippke

3793. Von Pippke.

Montag, 27.7.1812 ................... 289

Pischon, Friedrich August (1785-1857)

ab 1810 Hilfsprediger an der Dreifaltigkeitskirche Berlin

3819. An Friedrich August Pischon.

Berlin, Donnerstag, 31.12.1812 ............ 318

Pistorius, Charlotte (1777-1850)

geb. Pritzbuer, Dichterin, Frau des Pfarrers Johann Philipp Pistorius

3754. Von Luise von Willich, Friedrich Samuel Theodor Pritzbuer

und Charlotte Pistorius (auch an Henriette Schleier-

macher).

Poseritz und Garz, Sonntag, 1.3. (oder früher) bis

Sonntag, 8.3.1812 .................... 250 
3765. Von Luise von Willich und Charlotte Pistorius.

Poseritz, Montag, 30.3.1812

Marianne von Preußen (1785-1856)

geb. Prinzessin von Hessen-Homburg, Frau des Prinzen Wilhelm von Preußen (auch „Prinzessin Wilhelm“ genannt), Gegnerin Napoleons

3617. An Marianne von Homburg, Prinzessin von Preußen. Berlin, Montag, 8.4.1811 ............... 77

3622. Von Marianne von Homburg, Prinzessin von Preußen. Berlin, Donnerstag, 18.4.1811 .......... 83

Pritzbuer, Friedrich Samuel Theodor (1731-1819)

seit 1787 Propst in Garz auf Rügen, vorher Pfarrer in Reinkenhagen, Vater der Charlotte Pistorius

3754. Von Luise von Willich, Friedrich Samuel Theodor Pritzbuer und Charlotte Pistorius (auch an Henriette Schleiermacher).

Poseritz und Garz, Sonntag, 1.3. (oder früher) bis

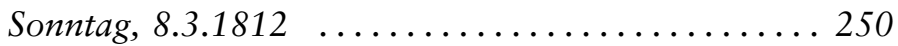

Reichardt, Luise (1779-1826)

Komponistin und Chorleiterin, Tochter des Johann Friedrich Reichardt aus erster Ehe

*3663. An Luise Reichardt.

Vor dem 19.8.1811 ................. 130

3664. Von Luise Reichardt.

Hamburg, Sonntag, 19.8.1811 ............. 131

3817. Von Luise Reichardt.

Hamburg, Sonnabend, 21.11.1812 ......... 315

Reil, Johann Christian (1759-1813)

Mediziner und Reformer, seit 1810 Professor der Medizin in Berlin *3579. An Johann Christian Reil.

Berlin, Freitag, 18.1. oder Sonnabend, 19.1.1811 . . 34

Reimer, Wilbelmine (1784-1864)

geb. Reinhardt, Frau des Verlegers und Schleiermacherfreundes Georg Andreas Reimer

*3875. Von Wilhelmine Reimer.

Potsdam, Mittwoch, 19.5. oder Donnerstag, 20.5.1813 
Röder, Wilbelm Carl Ferdinand von (1781-1813)

Adjutant Scharnhorsts, bei Kulm gefallen; Freund Schleiermachers

*3821. An Wilhelm von Röder.

Berlin, vor dem 9.1.1813 ............. 322

3822. Von Wilhelm von Röder.

Hirschberg, Sonnabend, 9.1.1813 .......... 322

*3824. An Wilhelm von Röder.

Berlin, wohl Sonntag, 24.1.1813 ........... 325

3831. Von Wilhelm von Röder.

Breslau, Sonnabend, 13.2.1813 ............. 329

*3887. Von Wilhelm von Röder.

Goldberg, vor dem 31.5.1813 ............. 421

*3893. An Wilhelm von Röder.

Berlin, Mittwoch, 2.6.1813 oder früher ........4424

*3900. An Wilhelm von Röder.

Berlin, Sonntag, 6.6. oder Montag, 7.6.1813 ..... 430

*3908. An Wilhelm von Röder.

Berlin, Sonnabend, 12.6.1813 .............. 439

*3927. An Wilhelm von Röder.

Berlin, vor dem 29.6.1813 ............. 473

Sack, Friedrich Samuel Gottfried (1738-1817)

Sobn des August Friedrich Wilhelm Sack, seit 1786 Oberkonsistorialrat, seit 1793 Oberhofprediger in Berlin

3705. Von Friedrich Samuel Gottfried Sack.

Berlin, Donnerstag, 21.11.1811 ........... 182

3772. Von Friedrich Samuel Gottfried Sack.

Berlin, Sonntag, 5.4.1812 _............. 267

Sack, Karl Heinrich (1789-1875)

Sohn des Friedrich Samuel Gottfried Sack, Enkel von Johann Joachim Spalding, Schüler Schleiermachers, 1813-15 im Krieg, 1815 im Domkandidatenstift, seit 1818 Professor der Theologie und Pfarrer in Bonn

3732. Von Karl Heinrich Sack.

Mitte Januar 1812 oder früher ............ 219 
Savigny, Friedrich Karl von (1779-1861)

Jurist, seit 1803 Privatdozent und Professor in Marburg, seit 1808 in Landshut und seit 1810 in Berlin

3771. An Friedrich Karl von Savigny.

Berlin, wohl März 1812

Scharnhorst, Gerhard Johann David von (1755-1813)

preußischer General und Militärreformer

*3834. An Gerhard von Scharnhorst.

Berlin, vor dem 8.3.1813 .............. 339

3835. Von Gerhard von Scharnhorst.

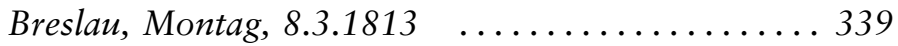

*3841. An Gerhard von Scharnhorst.

Berlin, vor dem 27.3.1813 ................ 351

Schlegel, August Wilhelm (1767-1845)

Schriftsteller, Übersetzer, Philologe, Bruder des Friedrich Schlegel

*3928. An August Wilhelm Schlegel.

Berlin, wohl Mai/Juni 1813

Schlegel, Friedrich (1772-1829)

Philosoph, Philologe und Dichter, Bruder des August Wilhelm Schlegel 3850. Von Friedrich Schlegel.

Wien, Donnerstag, 22.4.1813 ............ 359

3909. An Friedrich Schlegel.

Berlin, Sonnabend, 12.6.1813 ............. 439

Schleiermacher, Anne (Nanny) Maria Louise (1786-1869)

F. Schleiermachers Halbschwester, heiratet 1817 Ernst Moritz Arndt

3804. Von Anne (Nanny) Schleiermacher.

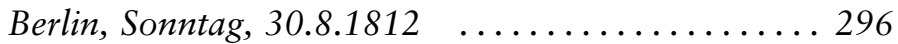

3806. Von Anne (Nanny) Schleiermacher.

Berlin, Mittwoch, 2.9.1812 .............. 297

3807. An Anne (Nanny) Schleiermacher

(auch von Luise von Willich).

Poseritz, Montag, 7.9.1812 ............. 298

3808. An Anne (Nanny) Schleiermacher.

Sagard, Sonntag, 13.9.1812 .............. 300

*3892. Von Anne (Nanny) Schleiermacher.

Vor dem 2.6.1813 ................. 424 
*3919. Von Anne (Nanny) Schleiermacher.

Vor dem 19.6.1813 .................... 464

*3920. An Anne (Nanny) Schleiermacher.

Berlin, Sonnabend, 19.6.1813 oder früher ...... 464

Schleiermacher, Johann Carl (Charles) (1772-1843)

Schleiermachers Bruder, Apotheker in Schmiedeberg

*3910. An Carl Schleiermacher.

Berlin, Sonnabend, 12.6.1813

Schleiermacher, Friederike Charlotte (Lotte) (1765-1831)

Erzieherin und Lehrerin in Gnadenfrei und Habendorf, F. Schleiermachers Schwester

3583. Von Charlotte (Lotte) Schleiermacher.

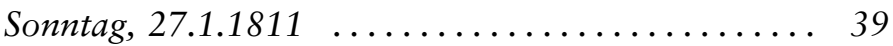

*3590. An Charlotte (Lotte) Schleiermacher.

Berlin, Sonnabend, 9.2.1811 ............ 46

3593. Von Charlotte (Lotte) Schleiermacher.

Gnadenfrei, Dienstag, 12.2.1811 .......... 51

*3609. An Charlotte (Lotte) Schleiermacher.

Berlin, vor dem 31.3.1811 ............. 72

3614. Von Charlotte (Lotte) Schleiermacher.

Gnadenfrei, Donnerstag, 4.4.1811 ......... 76

3631. Von Charlotte (Lotte) Schleiermacher.

Gnadenfrei, Dienstag, 14.5.1811 .......... 93

3638. Von Charlotte (Lotte) Schleiermacher.

Vor dem 29.5.1811 ................. 103

3639. Von Charlotte (Lotte) Schleiermacher.

Gnadenfrei, Mittwoch, 29.5.1811 .......... 104

3647. Von Charlotte (Lotte) Schleiermacher.

Vor dem 28.6.1811 ................. 111

3648. Von Charlotte (Lotte) Schleiermacher.

Gnadenfrei, Freitag, 28.6.1811 ........... 113

3653. Von Charlotte (Lotte) Schleiermacher.

Vor dem 16.7. bis Dienstag, 16.7.1811 ........ 120

3703. Von Charlotte (Lotte) Schleiermacher.

Gnadenfrei, Sonnabend, 16.11.1811 ......... 178

3706. Von Charlotte (Lotte) Schleiermacher.

Gnadenfrei, Donnerstag, 21.11.1811 ......... 184 
3719. Von Charlotte (Lotte) Schleiermacher.

Gnadenfrei, um den 20.12.1811 ............. 202

3721. Von Charlotte (Lotte) Schleiermacher.

Montag, 30.12.1811 bis Mittwoch, 1.1.1812 ..... 205

*3722. An Charlotte (Lotte) Schleiermacher.

Vor dem 1.1.1812 ....................... 207

3763. Von Charlotte (Lotte) Schleiermacher.

Gnadenfrei, Mittwoch, 25.3.1812 ............ 260

*3773. An Charlotte (Lotte) Schleiermacher.

Berlin, vor dem $12.4 .1812 \ldots \ldots \ldots \ldots \ldots . \ldots 268$

3774. Von Charlotte (Lotte) Schleiermacher.

Gnadenfrei, Sonntag, 12.4.1812 ............ 268

3788. Von Charlotte (Lotte) Schleiermacher.

Sonntag, 12.7.1812 .................... 281

*3799. An Charlotte (Lotte) Schleiermacher.

Berlin, Anfang August 1812 ................ 292

*3826. An Charlotte (Lotte) Schleiermacher.

Berlin, Januar 1813 .................... 326

3827. Von Charlotte (Lotte) Schleiermacher.

Gnadenfrei, wohl Anfang Februar bis Dienstag,

9.2.1813 .......................... 326

3840. Von Charlotte (Lotte) Schleiermacher

(auch an Henriette Schleiermacher).

Gnadenfrei, Donnerstag, 25.3.1813 ........... 349

*3845. An Charlotte (Lotte) Schleiermacher.

Anfang April 1813 ..................... 355

3852. Von Charlotte (Lotte) Schleiermacher.

Ende April 1813 ............................. 362

Schleiermacher, Henriette (Jette) (1788-1840)

geb. von Müblenfels, verw. von Willich, seit 1809 Friedrich Schleiermachers Frau

3683. Von Henriette Schleiermacher.

Berlin, Montag, 16.9. bis Dienstag, 17.9.1811 ... 149

3684. An Henriette Schleiermacher.

Hirschberg, Mittwoch, 18.9.1811 ............ 152

3686. An Henriette Schleiermacher.

Hirschberg, Sonnabend, 21.9.1811 ........... 154

3689. Von Henriette Schleiermacher.

Berlin, Montag, 23.9. bis Dienstag, 24.9.1811 ... 159 
3691. An Henriette Schleiermacher.

Breslau, Mittwoch, 25.9.1811 ............ 164

3692. An Henriette Schleiermacher.

Breslau, Freitag, 27.9. bis Sonnabend, 28.9.1811 . 166

3693. Von Henriette Schleiermacher.

Berlin, Freitag, 27.9. bis Sonnabend, 28.9.1811 .. 168

3858. An Henriette Schleiermacher.

Berlin, Donnerstag, 13.5. bis Sonnabend, 15.5.1813 ......................... 369

*3859. Von Henriette Schleiermacher.

Donnerstag, 13.5. oder Freitag, 14.5.1813 ...... 373

*3861. Von Henriette Schleiermacher.

Crossen, vor dem 18.5.1813 ............. 375

3870. An Henriette Schleiermacher.

Berlin, Sonntag, 16.5. bis Dienstag, 18.5.1813 . .. 377

3873. An Henriette Schleiermacher.

Berlin, Dienstag, 18.5. bis Donnerstag, 20.5.1813 . 383

*3874. Von Henriette Schleiermacher.

Schmiedeberg, Mittwoch, 19.5.1813 ........ 386

3877. An Henriette Schleiermacher.

Berlin, Donnerstag, 20.5. bis Sonnabend,

22.5 .1813 ......................... 387

3879. An Henriette Schleiermacher

Berlin, Sonnabend, 22.5. bis Dienstag, 25.5.1813 . . 393

*3880. Von Henriette Schleiermacher.

Schmiedeberg, Sonnabend, 22.5.1813 ......... 399

3882. An Henriette Schleiermacher.

Berlin, Dienstag, 25.5. bis Sonnabend, 29.5.1813 . . 401

3883. Von Henriette Schleiermacher.

Schmiedeberg, Mittwoch, 26.5.1813 .......... 409

3885. An Henriette Schleiermacher.

Berlin, Sonnabend, 29.5.1813 ............. 410

3886. An Henriette Schleiermacher.

Berlin, Sonntag, 30.5. bis Dienstag, 8.6.1813 ....4 411

3888. An Henriette Schleiermacher.

Berlin, Dienstag, 1.6.1813 ............. 421

3889. An Henriette Schleiermacher.

Berlin, Dienstag, 1.6.1813 .............. 422

*3890. An Henriette Schleiermacher.

Berlin, Dienstag, 1.6.1813 
3891. An Henriette Schleiermacher.

Berlin, Dienstag, 1.6.1813 ............... 423

3897. An Henriette Schleiermacher.

Berlin, Sonnabend, 5.6.1813 .............. 429

*3898. An Henriette Schleiermacher.

Berlin, Sonntag, 6.6.1813 ................ 429

*3899. Von Henriette Schleiermacher.

Schmiedeberg, Sonntag, 6.6.1813 ........... 430

3903. An Henriette Schleiermacher.

Berlin, Mittwoch, 9.6. bis Sonnabend, 12.6.1813 .. 433

*3911. Von Henriette Schleiermacher.

Schmiedeberg, Sonnabend, 12.6.1813 ......... 441

3912. An Henriette Schleiermacher.

Berlin, Sonntag, 13.6. bis Dienstag, 15.6.1813 ... 442

3915. Von Henriette Schleiermacher.

Schmiedeberg, Sonntag, 13.6. bis Mittwoch, 16.6.1813 .......................... 457

3916. An Henriette Schleiermacher.

Berlin, Dienstag, 15.6. bis Mittwoch, 16.6.1813 ... 459

3921. An Henriette Schleiermacher.

Berlin, Sonnabend, 19.6. bis Dienstag, 22.6.1813 .. 465

3922. Von Henriette Schleiermacher.

Schmiedeberg, Sonntag, 20.6.1813 ........... 467

3925. An Henriette Schleiermacher.

Berlin, Donnerstag, 24.6.1813 ............. 470

3926. An Henriette Schleiermacher.

Berlin, Sonnabend, 26.6.1813 .............. 472

Schlichtkrull, Adolf (1761-1835)

Präpositus in Poseritz, Mann der Sophie Schlichtkrull

*3672. An Adolf und Sophie Schlichtkrull.

Berlin, vor dem 27.8.1811 ................ 135

3685. Von Adolf Schlichtkrull.

Poseritz, Freitag, 20.9.1811 ................ 153

Schlichtkrull, Sophie (1766-1829)

geb. von Willich, Schwester Ehrenfried von Willichs

3573. Von Sophie Schlichtkrull.

Poseritz, Sonnabend, 12.1.1811 ............. 26 
*3672. An Sophie und Adolf Schlichtkrull.

Berlin, vor dem 27.8.1811 ............... 135

3718. Von Sophie Schlichtkrull.

Stralsund, Donnerstag, 19.12.1811 _.......... 201

Schmalz, Theodor Anton Heinrich (1760-1831)

Professor der Rechte in Berlin, erster Rektor der Berliner Universität 3604. Von Theodor Anton Heinrich Schmalz.

Berlin, Montag, 18.3.1811 ................ 64

Schulze, Johannes Karl Hartwig (1786-1869)

Theologe und Philologe, Schleiermacherhörer in Halle, 1808 Lehrer in Weimar, 1812 Direktor des Gymnasiums Hanau, 1813 Oberschulrat im Großherzogtum Frankfurt

3576. Von Johannes Karl Hartwig Schulze. Weimar, Dienstag, 15.1.1811 ............... 29

3680. An Johannes Karl Hartwig Schulze. Berlin, Freitag, 13.9.1811 ................ 147

3702. Von Johannes Karl Hartwig Schulze.

Weimar, Donnerstag, 14.11.1811 ............. 176

Sibbern, Frederick Christian (1785-1872)

dänischer Philosoph

3701. An Frederick Christian Sibbern.

Berlin, Montag, 14.11.1811 .............. 175

Simand

3640. Von Simand.

Mai 1811 .......................... 105

Stein, Heinrich Friedrich Karl Reichsfreiherr vom und zum (1757-1831) preußischer Staatsmann, bedeutender preußischer Reformer

3658. An Karl Freiherr vom und zum Stein.

Juli 1811 ........................ 126

3675. Von Karl Freiherr vom und zum Stein.

Juli/August 1811 ...................... 138 
Steffens, Henrich (1773-1845)

norwegischer Naturphilosoph, Schleiermacherfreund, ab 1811 Professor der Physik in Breslau

3569. Von Henrich Steffens.

Halle, Dienstag, $8.1 .1811 \ldots \ldots \ldots \ldots \ldots \ldots$

*3641. An Henrich Steffens.

Vor dem 1.6.1811. ................. 106

3642. Von Henrich Steffens.

Halle, Sonnabend, 1.6.1811 ............. 106

3661. Von Henrich Steffens.

Halle, Freitag, 9.8.1811 .............. 128

*3665. An Henrich Steffens.

Berlin, Mittwoch, 21.8.1811 ............... 132

3668. Von Henrich Steffens.

Halle, Sonntag, 25.8.1811 ............... 134

3711. Von Henrich und Johanna Steffens

(auch an Anne (Nanny) Schleiermacher).

Breslau, vor dem 10.12.1811 .............. 192

3715. Von Henrich Steffens.

Breslau, Mitte Dezember 1811 ............ 200

3753. Von Henrich und Johanna Steffens

(auch an Henriette und Anne (Nanny) Schleiermacher).

Breslau, Sonntag, 1.3. bis Montag, 2.3.1812 . . ... 247

\section{Steffens, Johanna (1785-1855)}

geb. Reichardt, Tochter von Johann Friedrich und Johanna Reichardt, seit 1803 Frau des Henrich Steffens

3605. Von Johanna Steffens.

Wohl Halle, Dienstag, 19.3.1811 ........... 65

3711. Von Johanna und Henrich Steffens

(auch an Anne (Nanny) Schleiermacher).

Breslau, vor dem 10.12.1811

3753. Von Johanna und Henrich Steffens

(auch an Henriette und Anne (Nanny) Schleiermacher).

Breslau, Sonntag, 1.3. bis Montag, 2.3.1812 .... 247

3849. Von Johanna Steffens

(auch an Anne (Nanny) Schleiermacher).

Breslau, Montag, 19.4.1813 ............. 357

*3865. An Johanna Steffens.

Vor dem 16.5.1813 ..................... 375 
3871. Von Johanna Steffens

(auch an Anne (Nanny) Schleiermacher).

Breslau, Sonntag, 16.5.1813 ................ 381

Twesten, August Detlev Christian (1789-1876)

Schleiermacherhörer in Berlin, Professor für systematische Theologie in Kiel ab 1814

3708. Von August Twesten.

Hamburg, Montag, 25.11.1811 ........... 186

3739. Von August Twesten.

Hamburg, Dienstag, 4.2.1812 ............ 233

3748. An August Twesten.

Berlin, Sonnabend, 22.2.1812 ............. 239

3750. Von August Twesten.

Hamburg, Freitag, 28.2.1812 ............ 245

3764. Von August Twesten.

Hamburg, Mittwoch, 25.3.1812 .......... 261

3790. Von August Twesten.

Eimsbüttel, Freitag, 17.7.1812 _......... 283

Veit, Simon (1754-1819)

Bankier in Berlin, erster Mann der Dorothea Schlegel (geschieden 1799)

*3907. Von Simon Veit.

Berlin, vor dem 12.6.1813 ..............4 438

Voß, Luise Sophie Caroline Gräfin von (1780-1865)

geb. von Berg, seit 1800 Frau des August Ernst Graf von Voß

*3704. Von Luise Sophie Caroline Gräfin von Voß.

Vor dem 21.11.1811 ................. 181

3709. An Luise Sophie Caroline Gräfin von Voß.

Berlin, Sonnabend, 30.11.1811 ............. 188

*3862. Von Luise Sophie Caroline Gräfin von Voß.

Freitag, 14.5. oder Sonnabend, 15.5.1813 ...... 375

*3867. Von Luise Sophie Caroline Gräfin von Voß.

Sonnabend, 15.5. oder Sonntag, 16.5.1813 ..... 376

*3876. Von Luise Sophie Caroline Gräfin von Voß.

Donnerstag, 20.5.1813 oder früher .......... 387

3894. An Luise Sophie Caroline Gräfin von Voß.

Berlin, Mittwoch, 2.6.1813 ............ 425 
LXVI Verzeichnis der einzelnen Briefwechsel

3901. An Luise Sophie Charlotte Gräfin von Voß.

Berlin, Montag, 7.6.1813 ............... 430

*3902. Von Luise Sophie Charlotte Gräfin von Voß.

Berlin, Mittwoch, 9.6.1813 ............ 432

Weinlig, J.

3746. Von J. Weinlig.

Wohl Donnerstag, 20.2.1812 (oder am 20.2. eines

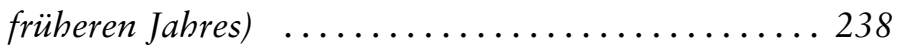

Wiggers, Gustav Friedrich (1777-1860)

seit 1810 Professor der Theologie und Direktor des pädagogischen Seminars in Rostock

3727. Von Gustav Friedrich Wiggers.

Rostock, Donnerstag, 2.1.1812 _......... 210

Willich, Ernst Heinrich Brandanus von (1755-1826)

Sohn des Philipp Georg von Willich aus einer früheren Ehe, Postmeister und Oberstleutnant

3563. Von Ernst Heinrich Brandanus von Willich.

Bunzlau, Freitag, 4.1.1811 .............. 4

*3616. An Ernst Heinrich Brandanus von Willich.

Berlin, Sonnabend, 6.4.1811 .............. 77

*3681. An Ernst Heinrich Brandanus von Willich.

Görlitz, Montag, 16.9.1811 ................. 149

3872. Von Ernst Heinrich Brandanus von Willich.

Bunzlau, Sonntag, 16.5.1813

Willich, Heinrich Christoph von (1759-1827)

Sohn Philipp Georg und Marianne Regina von Willichs, Pastor in Sagard auf Rügen

3571. Von Heinrich Christoph von Willich (auch an Henriette Schleiermacher).

Sagard, Freitag, 11.1.1811 ............ 22

*3623. An Heinrich Christoph von Willich.

Berlin, vor dem 20.4.1811 ............... 83

*3670. An Heinrich Christoph von Willich.

Berlin, vor dem 26.8.1811 ............. 135 
3673. Von Heinrich Christoph von Willich

(auch an Luise von Willich).

Sagard, Dienstag, 27.8.1811.

*3707. An Heinrich Christoph von Willich.

Berlin, Montag, 25.11.1811 ............... 186

3712. Von Heinrich Christoph von Willich.

Stralsund, Dienstag, 10.12.1811 ............. 195

3801. Von Heinrich Christoph von Willich.

Sagard, Dienstag, 11.8.1812 ............... 292

3814. Von Heinrich Christoph von Willich.

Sagard, Dienstag, 17.11.1812 .............. 310

*3830. Von Heinrich Christoph von Willich

(auch an Henriette Schleiermacher).

Sagard, um den 9.2.1813 ................. 329

3844. Von Heinrich Christoph und Doris von Willich.

Sagard, Dienstag, 6.4.1813 ............... 353

\section{Willich, Margarethe Dorothea (Doris) von}

geb. Bokelmann, verw. Simon, dritte Ehefrau des Heinrich Christoph von Willich

3844. Von Doris und Heinrich Christoph von Willich.

Sagard, Dienstag, 6.4.1813

\section{Willich, Maria Christiane Luise von (1767-1849)}

Schwester des Feldpredigers Ehrenfried von Willich

3687. Von Luise von Willich.

Bunzlau, Sonnabend, 21.9.1811 ............ 156

3690. Von Luise von Willich.

Bunzlau, Dienstag, 24.9.1811 .............. 162

3720. An Luise von Willich.

Berlin, Donnerstag, 26.12.1811 ............. 204

3729. Von Luise von Willich.

Poseritz, Sonntag, 12.1.1812 bis Montag,

13.1 .1812 ......................... 213

3737. Von Luise von Willich.

Poseritz und Garz, Donnerstag, 30.1. bis Montag, 17.2.1812 ........................... 221

3747. Von Luise von Willich.

Wohl Anfang 1812 ....................... 239 
LXVIII Verzeichnis der einzelnen Briefwechsel

3754. Von Luise von Willich, Friedrich Samuel Theodor Pritzbuer und Charlotte Pistorius (auch an Henriette Schleiermacher).

Poseritz und Garz, Sonntag, 1.3. (oder früher) bis Sonntag, 8.3.1812 .................. 250

3755. An Luise von Willich.

Berlin, Sonntag, 8.3.1812 .............. 254

3765. Von Luise von Willich und Charlotte Pistorius.

Poseritz, Montag, 30.3.1812 ............ 263

*3800. Von Luise von Willich und Charlotte von Kathen.

Vor dem 11.8 .1812 ................. 292

3802. Von Luise von Willich.

Sagard, Mittwoch, 12.8. bis Donnerstag, 13.8.1812 293

*3815. Von Luise von Willich.

Sagard, Sonnabend, 21.11.1812 oder früber .....313

3832. Von Luise von Willich.

Sagard und Poseritz, Dienstag, 16.2.1813 bis späte-

stens Anfang April 1813 ................. 333

3881. An Luise von Willich.

Berlin, Sonntag, 23.5.1813 .............. 400

3884. Von Luise von Willich.

Vor dem 27.5.1813 ..................... 409

3906. Von Luise von Willich.

Götemitz, wohl um den 10.6.1813 ..........448

3914. Von Luise von Willich.

Poseritz, Sonntag, 13.6. bis Dienstag, 15.6.1813 ... 447

3924. Von Luise von Willich.

Poseritz, Mittwoch, 23.6. bis Donnerstag,

24.6.1813

Willich, Marianne von (geb. 1760)

Schwester des Heinrich Christoph von Willich, später verheiratete Dreist

3843. Von Marianne von Willich.

Sagard, Sonntag, 4.4. bis Montag, 5.4.1813 ..... 351

Wolf, Christian Wilhelm Friedrich August (1759-1824)

Altphilologe, seit 1810 Professor in Berlin

3792. Wohl an Friedrich August Wolf.

Berlin, Mittwoch, 22.7.1812 ........... 288 
Wolf(f), Philipp Wilbelm (1766-1822)

Theologe, seit 1810 Superintendent in Zossen

3578. Von Philipp Wilhelm und Ena (Julie) Wolf

(diese auch an Henriette Schleiermacher).

Zossen, Donnerstag, 17.1.1811 .......... 32

3756. Von Philipp Wilhelm Wolf.

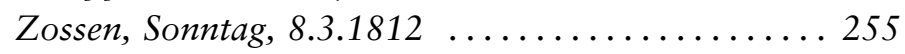

Wolf(f), Ena (Julie)

Frau des Philipp Wilhelm Wolf(f)

3578. Von Philipp Wilhelm und Ena (Julie) Wolf

(diese auch an Henriette Schleiermacher).

Zossen, Donnerstag, 17.1.1811 .......... 32

Wolfart, Karl Christian (1778-1832)

Mediziner, seit 1810 Dozent, später Professor an der Berliner Universität

*3869. An Karl Christian Wolfart.

Berlin, Sonntag, 16.5.1813 ............ 376

Zenker, Johann Gottlieb Friedrich (1753-1826)

Geheimer Kriegsrat und Hof-und Staatsrentmeister in Berlin

3612. Von Johann Gottlieb Friedrich Zenker.

Berlin, Montag, 1.4.1811 ............. 75

\section{Briefwechsel mit nicht ermittelten Personen}

3600. An Unbekannt.

Berlin, Mittwoch, 13.3.1811 ............ 61

3632. Von Unbekannt.

Mitte Mai 1811 oder früher ............. 95

3644. Von Unbekannt.

Anfang Juni 1811 oder früber ............. 108

3655. Von Unbekannt.

Berlin, Montag, 22.7.1811 ............. 123

3662. Von Unbekannt.

Juni bis Mitte August 1811 ............... 130

3700. Von Unbekannt (Weinhändler).

Anfang November 1811 oder früher ......... 175 
*3723. An Unbekannt (einen Geistlichen).

1811 ............................... 207

3742. An Unbekannt.

Mitte Februar 1812 oder früher ............. 236

3743. Von Unbekannt.

Mitte Februar 1812 oder früher ............. 237

3744. Von Unbekannt.

Mitte Februar 1812 oder früher .............. 237

3751. Von Unbekannt.

Februar 1812 oder früher .................. 246

3752. Von Unbekannt.

Februar 1812 oder früher ................. 246

3760. Von Unbekannt.

Vor dem 20.3 .1812 .................... 259

3761. Von Unbekannt.

Vor dem 20.3 .1812 .................... 260

3762. Von Unbekannt.

Sonnabend, 21.3.1812 .................. 260

*3766. Von Unbekannt („Nestor“).

Berlin, März 1812 ...................... 265

*3767. An Unbekannt („Nestor").

Berlin, März 1812 ...................... 265

*3769. Von Unbekannt („Nestor“).

Berlin, März 1812 ..................... 265

3780. Von Unbekannt.

Vor dem 30.4 .1812 .................... 274

3781. Von Unbekannt.

Anfang Mai 1812 oder früher ............... 274

3784. Von Unbekannt.

Ende Juni 1812 oder früher ................ 276

3803. Von Unbekannt.

Vor dem 18.8 .1812 ...................... 295

3805. An Unbekannt.

August 1812 ......................... 297 


\section{Amtlicher Briefwechsel}

\section{Schleiermachers Tätigkeit an der Dreifaltigkeitskirche}

3611. Von Carl Friedrich Grabl.

Berlin, Montag, 1.4.1811 ................ 74

3674. An Andreas Jakob Hecker.

Berlin, Sonnabend, 31.8.1811 ............ 137

*3726. Von Friedrich Herzberg.

Berlin, Mittwoch, 1.1.1812 .............. 210

3730. An Friedrich Herzberg (auch von der Sektion des Unterrichts).

Berlin, Montag, 13.1.1812 ............... 217

3735. An Andreas Jakob Hecker.

Berlin, Sonnabend, 25.1.1812 ............ 220

3775. Von Gottfried Mieth.

Berlin, Montag, 13.4.1812 ............. 270

3778. An die Kirchenvorsteher der Dreifaltigkeitsgemeinde.

Berlin, Freitag, 24.4.1812 ............. 272

3819. An Friedrich August Pischon.

Berlin, Donnerstag, 31.12.1812 ............. 318

\section{Schleiermachers Tätigkeit an der Universität}

Theologische Fakultät

3629. An die Theologische Fakultät.

Berlin, Mittwoch, 1.5.1811 ............. 89

3659. An die Theologische Fakultät.

Berlin, Donnerstag, 1.8.1811 ........... 127

Schleiermachers Tätigkeit in der Sektion des öffentlichen Unterrichts

Direktion des Landschullehrerseminars

3594. An die Direktion des Landschullehrerseminars

(auch von der Sektion des öffentlichen Unterrichts).

Berlin, Mittwoch, 20.2.1811 ........... 53 
LXXII Verzeichnis der einzelnen Briefwechsel

Hardenberg, Karl August Freiberr von (in seiner Funktion als Staatskanzler)

3731. An Karl August Freiherr von Hardenberg

(auch von der Sektion des öffentlichen Unterrichts).

Berlin, Montag, 13.1.1812 .............. 218

3813. An Karl August Freiherr von Hardenberg

(auch von der Sektion des öffentlichen Unterrichts).

Berlin, Montag, 9.11.1812 ............. 308

Geistliche und Schuldeputation der Kurmärkischen Regierung

3779. An die Geistliche und Schuldeputation der Kurmärkischen

Regierung

(auch von der Sektion des öffentlichen Unterrichts).

Berlin, Montag, 27.4.1812 .............. 273

3798. An die Geistliche und Schuldeputation der KurMärkischen

Regierung

(auch von der Sektion des öffentlichen Unterrichts).

Berlin, Sonntag, 9.8.1812................. 291 